\title{
Identification of thrombomodulin as a dynamic monitoring biomarker for deep venous thrombosis evolution
}

\author{
XI CHENG ${ }^{1,2^{*}}$, BAOLAN SUN $^{1 *}$, SHIYI LIU $^{1,2}$, DANDAN LI $^{1}$, XIAOQING YANG $^{3}$ and YUQUAN ZHANG ${ }^{3}$ \\ ${ }^{1}$ Medical College, Nantong University; ${ }^{2}$ Laboratory Animal Center, Nantong University; \\ ${ }^{3}$ Department of Gynecology and Obstetrics, Affiliated Hospital of Nantong University, Nantong, Jiangsu 226001, P.R. China
}

Received July 23, 2020; Accepted October 26, 2020

DOI: $10.3892 /$ etm.2020.9574

\begin{abstract}
It has been demonstrated that thrombomodulin (TM) serves an important role in the formation of deep venous thrombosis (DVT) and is regarded to be a marker that can be used to measure vascular endothelial cell damage. However, how TM levels change during DVT evolution has not yet been well understood. The current study aimed to investigate the dynamic changes of TM during the evolution of DVT and explore the possible mechanisms behind these. A total of 48 patients newly diagnosed with DVT and 23 matched healthy controls were enrolled in the present study, and their plasma TM levels were examined and compared. In addition, a DVT model was established using Sprague-Dawley rats via the 'stenosis' method. The thrombi size, histopathologic changes and expression of $\mathrm{TM}$ and $\mathrm{NF}-\kappa \mathrm{B}$ in plasma and venous endothelium were measured at 9 different time points $(1,4$, 6, 12 and $24 \mathrm{~h}$, and at 3, 7, 14 and 21 days). Finally, the effect of inhibiting the activation of NF- $\mathrm{BB}$ on TM was investigated using pyrrolidine dithiocarbamate (PDTC), which is a potent inhibitor of the NF- $\mathrm{B}$ pathway. The results of the current study indicated that the mean level of plasma TM in patients with DVT was significantly increased compared with healthy controls. In addition, thrombi size (clot length and weight), TM and NF- $\kappa \mathrm{B}$ expression in the animal model plasma exhibited three distinct periods (1-12, 24 h-day 7 and 14-21) of markedly different results between periods. Immunofluorescence results confirmed the co-localization of TM and NF- $\kappa \mathrm{B}$ in endothelial cells. In addition, it was indicated that the expression of TM in the endothelium of DVT models was upregulated compared with the control, while $\mathrm{NF}-\kappa \mathrm{B}$ was significantly downregulated. Following the administration of PDTC, the level
\end{abstract}

Correspondence to: Dr Yuquan Zhang, Department of Gynecology and Obstetrics, Affiliated Hospital of Nantong University, 20 Xisi Road, Nantong, Jiangsu 226001, P.R. China

E-mail: jsnt_zhangyuquan@163.com

${ }^{*}$ Contributed equally

Key words: thrombomodulin, nuclear factor- $\mathrm{B}$, deep venous thrombosis, diagnosis, biomarker of $\mathrm{NF}-\kappa \mathrm{B}$ and $\mathrm{TM}$ in the plasma were decreased significantly dose-dependently. The results of the current study suggested that TM was involved in the evolution of DVT and may be used as a dynamic biomarker to measure disease activity. Furthermore, the expression of TM during the evolution of DVT was indicated to be associated with the NF- $\mathrm{BB}$ signaling pathway.

\section{Introduction}

Venous thromboembolism (VTE), including deep venous thrombosis (DVT) and pulmonary embolism (PE), is the third most common cardiovascular disease following myocardial infarction and stroke, with $\sim 10$ million new cases diagnosed worldwide each year (1). VTE is associated with a relatively high mortality rate and in a 25-year (1966-1990) inception cohort $(n=2218)$ of Olmsted County, Minnesota, $6 \%$ of patients with DVT and $10 \%$ of patients with PE died within 30 days after disease onset (2). The long-term morbidity and mortality related to VTE mainly results from complications such as post-thrombotic syndrome, recurrent venous thromboembolism and chronic thromboembolic pulmonary hypertension (3). Due to its nonspecific clinical manifestations, the misdiagnosis and omission rate of VTE is relatively high (1). It is important to examine the dynamic changes of thrombus and the underlying molecular mechanisms of VTE in order to provide patients with a diagnosis and treatment strategies.

Previous studies have revealed that risk factors such as hypercoagulability, stasis, vascular wall damage or dysfunction may increase the risk of VTE (1). Thrombomodulin (TM), which is a specific molecular marker for vascular endothelial cell damage, is a transmembrane glycoprotein that exists on the surface of vascular endothelial cells and serves as a cofactor for thrombin-mediated activation of protein $\mathrm{C}$ (PC), which is a major anticoagulant that downregulates thrombin formation and decreases thrombus formation $(4,5)$. Elevated TM levels can be detected in different types of body fluid in a variety of diseases associated with endothelial cell injuries (4). In a previous study, plasma TM levels were reported to be increased in patients with VTE and were positively correlated with Caprini score (6). Furthermore, familial TM deficiency has been linked to the most common inherited thrombotic disorders (7). TM has also been reported to be associated with 
the formation of DVT; however, few reports have examined the dynamic changes of TM during the evolution of DVT.

The increased expression of TM commonly occurs during inflammation and tumor development, both of which are closely associated with the NF- $\mathrm{B}$ signaling pathway $(8,9)$. Increased TM expression has been observed in bladder cancer, pancreatic cancer, prostate cancer and sepsis, and has been demonstrated to be closely associated with the activation of $\mathrm{NF}-\kappa \mathrm{B}(10-14)$. Previous studies have suggested that the occurrence of DVT is closely associated with inflammation (15), and is regulated via the Sirtuin-1/NF- $\kappa \mathrm{B}$ signaling pathway (16). However, to the best of our knowledge, whether TM is also regulated by $\mathrm{NF}-\kappa \mathrm{B}$ during the evolution of DVT has yet to be determined.

The current study aimed to examine the expression of TM during the evolution of DVT and to explore its potential association with the NF- $\kappa \mathrm{B}$ pathway using pyrrolidine dithiocarbamate (PDTC), which is an inhibitor of NF- $\kappa \mathrm{B}(17,18)$. The current study also aimed to identify novel diagnosis and treatment strategies that could be used in patients with DVT.

\section{Materials and methods}

Patients. A total of 48 consecutive patients diagnosed with DVT at the Affiliated Hospital of Nantong University (Nantong, Jiangsu, China) from April 2019 to December 2019 were enrolled in the present study. The levels of TM of DVT patients were analyzed in the 3 age groups, which were defined as the $Y$ group $(<64$ years; $n=26), M$ group $(65-74$ years; $n=11)$ and the $\mathrm{O}$ group $(>75$ years; $n=10)$. Patients with any acute or chronic infection or cancer-related diseases were excluded. Additionally, a total of 23 age and sex matched healthy individuals were enrolled at Affiliated Hospital of Nantong University (Nantong, Jiangsu, China) from May 2019 to September 2019 as controls in the current study. Venous blood ( $2 \mathrm{ml}$ ) was drawn from the patients with DVT and healthy controls, and collected in sterile tubes. The present study was approved by the Ethics Committee of Affiliated Hospital of Nantong University, and all participants were required to provide their written informed consent before enrollment. Clinical characteristics of patients in these two groups are summarized in Table I.

Experimental animals and grouping. Sexually mature male Sprague-Dawley rats (weight, 300 $\pm 20 \mathrm{~g} ; \mathrm{n}=76$ ) that were 10-12 weeks of age, were purchased from the Laboratory Animal Center of Nantong University. The rats were kept under a $12 \mathrm{~h}$ light/dark cycle, a temperature of $23 \pm 3^{\circ} \mathrm{C}$ and a humidity of 55-60\%, and had free access to chow and water. All protocols were approved by the institutional review board for animal experiments at Nantong University. A total of 9 different time points of the DVT model group (1, 4, 6, 12 and $24 \mathrm{~h}$, and at 3, 7, 14 and 21 days) and a control group were used to investigate the expression of $\mathrm{TM}$ and $\mathrm{NF}-\kappa \mathrm{B}$ during the evolution of DVT ( $n=6 \times 10)$. In order to further investigate the underlying $\mathrm{NF}-\kappa \mathrm{B}$ pathway, another 16 rats were divided randomly into 4 groups: i) DVT model group $(0 \mathrm{mg} / \mathrm{kg}$ PDTC); ii) DVT rats treated with $50 \mathrm{mg} / \mathrm{kg}$ PDTC; iii) DVT rats treated with $100 \mathrm{mg} / \mathrm{kg}$ PDTC; iv) DVT rats treated with $150 \mathrm{mg} / \mathrm{kg}$ PDTC, $\mathrm{n}=4 \times 4$ ).
DVT animal model. Inferior vena cava (IVC) stenosis procedures were performed as described previously under aseptic conditions to establish the DVT model $(16,19)$. Specifically, $90 \%$ ligation of IVC and complete ligation of side branches were performed in order to recapitulate human DVT (20) (Fig. S1). The control animals only received dissection of IVC and side branches without vein ligation. Anesthesia was induced in an isoflurane chamber (3-4\%) and maintained with a face mask using 1-2\% isoflurane. The rats were allowed to recover post-surgery and housed under a $12 \mathrm{~h}$ light/dark cycle, a temperature of $23 \pm 3^{\circ} \mathrm{C}$ and a humidity of $55-60 \%$, and had free access to chow and water during recovery. Finally, the rats were sacrificed at h 1, 4, 6 and 12 and day 1, 3, 7, 14 and 21 post operation, and the control animals were sacrificed at a median time point of day 1 post surgery. A total of $2 \mathrm{ml}$ venous blood was collected via the apex cordis into EDTA-coated capillary tubes prior to the rats being sacrificed. All rats were sacrificed by cervical dislocation under deep anesthesia with $2 \%$ isoflurane. Post-sacrifice, the thrombosed IVC was carefully dissected on ice, while the weight $(\mathrm{G})$ and length $(\mathrm{cm})$ of thrombi harvested from the IVC were measured with an electronic balance (Shanghai Mettler Toledo Co., Ltd.) and vernier caliper (Deli Co., Ltd.). Thrombi and endothelial tissues were soaked in paraformaldehyde and used for hematoxylin and eosin $(\mathrm{H} \& \mathrm{E})$ staining and immunofluorescence staining. The remaining tissues were stored at $-80^{\circ} \mathrm{C}$ and subsequently used for western blot analysis.

Digital subtraction angiography (DSA). Venography was performed on day 1 after receiving IVC-ligations or sham operations. A fluoroscopy unit (Shimadzu type C-VISION PLUS; Philips Medical Systems, Inc.) was used to estimate the thrombus mass within the IVC after ligation. Inhalant isoflurane induced in an isoflurane chamber (3-4\%) and maintained with a face mask using 1-2\% isoflurane was used for the induction and maintenance of anesthesia on day 1 post operation. Rats were placed on supine position under the X-ray source, and $2 \mathrm{ml}$ of contrast medium (Iohexol; OMNIPAQUE 350; GE Healthcare) was injected through the tail vein at a rate of $0.3 \mathrm{ml} / \mathrm{sec}$ to visualize the adequate filling of the IVC (21). The venographic images were acquired at 6 frames/sec. The length and width of clots were measured using interventional workspot (Allura 3D-RA Release 5; Philips Medical Healthcare, Inc.).

ELISA. Venous blood collected from humans and rats was centrifugated at $1,789 \times \mathrm{g}$ at $4^{\circ} \mathrm{C}$ for $10 \mathrm{~min}$ and the plasma was collected and stored at $-80^{\circ} \mathrm{C}$ until subsequent use. Plasma levels of TM (Human TM ELISA kit, cat. no. AE90697Hu; Rat TM ELISA kit; cat. no. AE90697Ra) and NF- $\kappa$ B (Rat nuclear factor Kappa B ELISA kit; cat. no. AE91992Ra) was quantified using an ELISA kit (Shanghai United Biotech Co., Ltd.) according to manufacturer's protocol. All samples were repeated in triplicate.

Histopathological analysis. The thrombotic and endothelial tissues of control rats and rats sacrificed at each of the nine time-points were fixed in $4 \%$ paraformaldehyde overnight at room temperature and processed as 4-6 $\mu \mathrm{m}$ thick paraffin sections, then stained with H\&E (20 min in hematoxylin solution and $60 \mathrm{sec}$ in eosin solution at room temperature) 
Table I. Baseline characteristics of patients in the DVT and control group.

\begin{tabular}{lccc}
\hline Characteristic & $\begin{array}{c}\text { Control } \\
(\mathrm{N}=23)\end{array}$ & $\begin{array}{c}\mathrm{DVT} \\
(\mathrm{N}=48)\end{array}$ & P-value \\
\hline Age, years & $52.77 \pm 13.93$ & $59.68 \pm 18.16$ & 0.119 \\
Sex, male & 11 & 21 & 0.802 \\
Body mass index, $\mathrm{kg} / \mathrm{m}^{2}$ & $24.33 \pm 3.48$ & $24.50 \pm 3.29$ & 0.849 \\
Diabetes & 0 & 10 & 0.02 \\
Hypertension & 0 & 15 & 0.002 \\
Smoking & 0 & 0 & - \\
Malignant disease & 0 & 0 & - \\
Recent surgery or & 0 & 0 & - \\
immobilization & & & \\
VTE history & 0 & 0 & - \\
\hline
\end{tabular}

DVT, deep venous thrombosis, VTE, venous thromboembolism.

following standard procedures. Light microscopy (magnification, $\mathrm{x} 4$ ) was used to observe typical pathological changes in rat inferior vena cava thrombus at different time points.

Immunofluorescence. The thrombotic and endothelial tissues on day 1 post surgery were fixed in $4 \%$ paraformaldehyde overnight at room temperature and sliced as 4-6 $\mu \mathrm{m}$ thick paraffin sections. Immunofluorescence staining was performed on paraffin embedded tissue sections to detect the distribution of TM and NF- $\mathrm{KB}$ (p65). The slices were blocked with 5\% bovine Serum Albumin (Sigma-Aldrich; Merck KGaA) for $2 \mathrm{~h}$ at room temperature and incubated independently with different primary antibodies at $4^{\circ} \mathrm{C}$ overnight. Anti-TM antibodies (rabbit polyclonal; 1:600; Mybiosource, Inc; cat. no. MBS9606019) and anti-p65 (rabbit polyclonal; 1:1,000; ProteinTech Group, Inc; cat. no. 10745-1-AP) were used as primary antibodies. CD34 was used as a marker to define the distribution of vascular endothelial cell (CD34 mouse monoclonal; 1:400; NOVUS Biologicals, LLC; cat. no. SA00003-1).

Western blot analysis. Protein was extracted from thrombus and venous endothelium tissues of control rats and DVT rats at nine time-points by dissolving in a lysis buffer using a Tissue or Cell Total Protein Extraction kit (Nanjing KeyGen Biotech Co., Ltd.) following manufacturer's instructions. The concentration of protein was quantified using a Drop One spectrophotometer (Thermo Fisher Scientific, Inc.). $50 \mu \mathrm{g}$ total protein per gel lane was then electrophoretically separated on $10 \%$ polyacrylamide gels and transferred onto nitrocellulose membranes. After blocking with 5\% non-fat milk for $1.5 \mathrm{~h}$ at room temprature, blots were incubated with anti-TM (1:1,000; rabbit anti-rat TM; Abcam; cat. no. ab187075.), anti-p65 (1:1,000; rabbit anti-rat p65, Cell Signaling Technology, Inc; cat. no. $8242 \mathrm{~S}$.) overnight at $4^{\circ} \mathrm{C}$. Membranes were then were rinsed six times with $1 \mathrm{x}$ tris buffered saline with $0.1 \%$ Tween (TBST), before incubation with a secondary antibody (1:10,000; goat anti-rabbit IgG; Absin Bioscience, Inc; cat. no. abs20040ss.) for $2 \mathrm{~h}$ at room temperature. After rinsing six times for $10 \mathrm{~min}$ each time, images were acquired using a Tanon 2500 Gel Imaging System (Yph-Bio Co., Ltd.) and processed using ImageJ software (v.1.8.0; National Institutes of Health). $\beta$-actin was used as the loading control.

Administration of PDTC. PDTC (Selleck Chemicals) was dissolved in sterilized water and injected at a concentration of $25 \mathrm{mg} / \mathrm{ml}$, similar to a previously reported protocol (17). We defined the four groups as follows: C, DVT model group (0 mg/kg PDTC); LI, DVT rats treated with $50 \mathrm{mg} / \mathrm{kg}$ PDTC; MI, DVT rats treated with $100 \mathrm{ml} / \mathrm{kg}$ PDTC; HI, DVT rats treated with $150 \mathrm{mg} / \mathrm{kg}$ PDTC. A total of 4 different concentrations $100 \mathrm{ml} / \mathrm{kg}$ of PDTC were injected into $100 \mathrm{ml} / \mathrm{kg}$ the PDTC groups and DVT model group respectively at $6 \mathrm{~h}$, day 1,2 and 3 post operation via the tail vein at a rate of $0.05-0.1 \mathrm{ml} / \mathrm{sec}$. Blood samples were taken $6 \mathrm{~h}$ following each administration via retro-orbital sinus as previously reported to detect the expression of TM and NF- $\mathrm{kB}$ in plasma $(22,23)$.

Statistical analysis. Statistical analysis was performed using the statistical software package SPSS (Version 16.0; SPSS, Inc.), and the graphs were generated using GraphPad Prism 6.0 (GraphPad Software, Inc.). One-way ANOVA followed by the Bonferroni post-hoc test was used to compare differences of age and body mass index between the DVT group and the control group. Differences in categorical variables between groups were tested using the $\chi^{2}$ or Fisher's exact test. One-way ANOVA followed by the Bonferroni post hoc test was also used to compare differences between multiple groups. Two-way ANOVA followed by the Bonferroni post hoc test was only used to compare differences between different time points in the PDTC groups and DVT model group. Receiver operating characteristic (ROC) curves and the area under the ROC curve (AUC) were used to compare the sensitivity and specificity of the plasma levels of TM for the diagnosis of DVT. All data were expressed as mean $\pm \mathrm{SEM}$, and $\mathrm{P}<0.05$ was considered to indicate a statistically significant difference.

\section{Results}

The level of TM is upregulated in patients with DVT. The levels of TM in plasma collected from 48 patients with DVT and 23 healthy controls was measured using ELISA. Clinical characteristics of patients in these two groups are summarized in Table I. Compared with the control group $(8.43 \pm 0.29 \mu \mathrm{g} / \mathrm{l})$, plasma TM levels were significantly increased in patients with DVT (10.46 $\pm 0.17 \mu \mathrm{g} / 1 ; \mathrm{P}<0.01$; Fig. 1A). The results also indicated that the levels of TM in the O group $(11.20 \pm 0.94 \mu \mathrm{g} / \mathrm{l})$ were significantly higher compared with the Y $(10.36 \pm 1.05 \mu \mathrm{g} / \mathrm{l}$; $\mathrm{P}<0.05)$ and $\mathrm{M}$ group $(10.23 \pm 1.27) \mu \mathrm{g} / \mathrm{l} ; \mathrm{P}<0.05$; Fig. $1 \mathrm{~B})$. ROC curve analysis for TM was performed and the AUC value ranged from 0.795 to 0.953 for TM in identifying patients with DVT from healthy controls (Fig. 1C). The results suggested that TM level could differentiate patients with DVT from non-thrombotic controls with an AUC of 0.874 , and the best cut off value for predicting DVT was $10.12 \mu \mathrm{g} / \mathrm{l}$.

Visualizing venous thrombosis by DSA. DSA was performed to ensure the successful establishment of thrombosis in the IVC. Anesthetized rats were monitored by continuous camera recording. Fig. 2 presents the images at different time points 
A

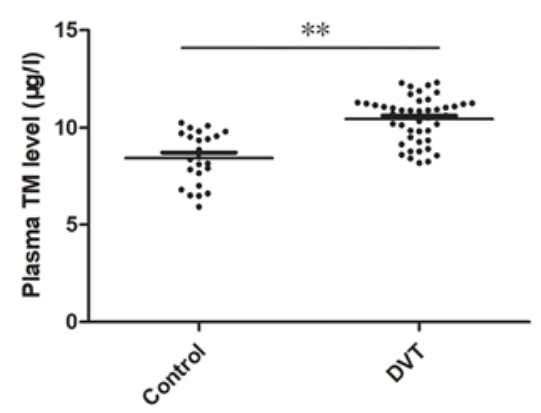

B

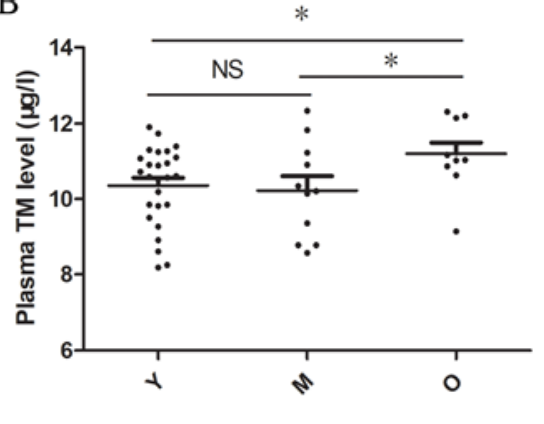

$\mathrm{C}$

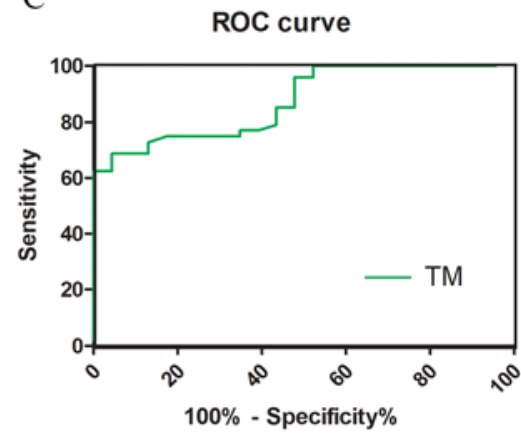

Figure 1. TM is upregulated in patients with DVT. (A) Upregulation of TM in patients with DVT compared with healthy controls. (B) The plasma TM levels of patients with DVT in the 3 age groups. Y group, $<64$ years; M group, $65-74$ years; O group, $>75$ years. (C) ROC curves showing the area under the ROC curve. Data are presented as means $\pm \mathrm{SD}$. ${ }^{*} \mathrm{P}<0.05,{ }^{* *} \mathrm{P}<0.01$. ROC, Receiver operating characteristic; TM, Thrombomodulin; DVT, deep vein thrombosis.

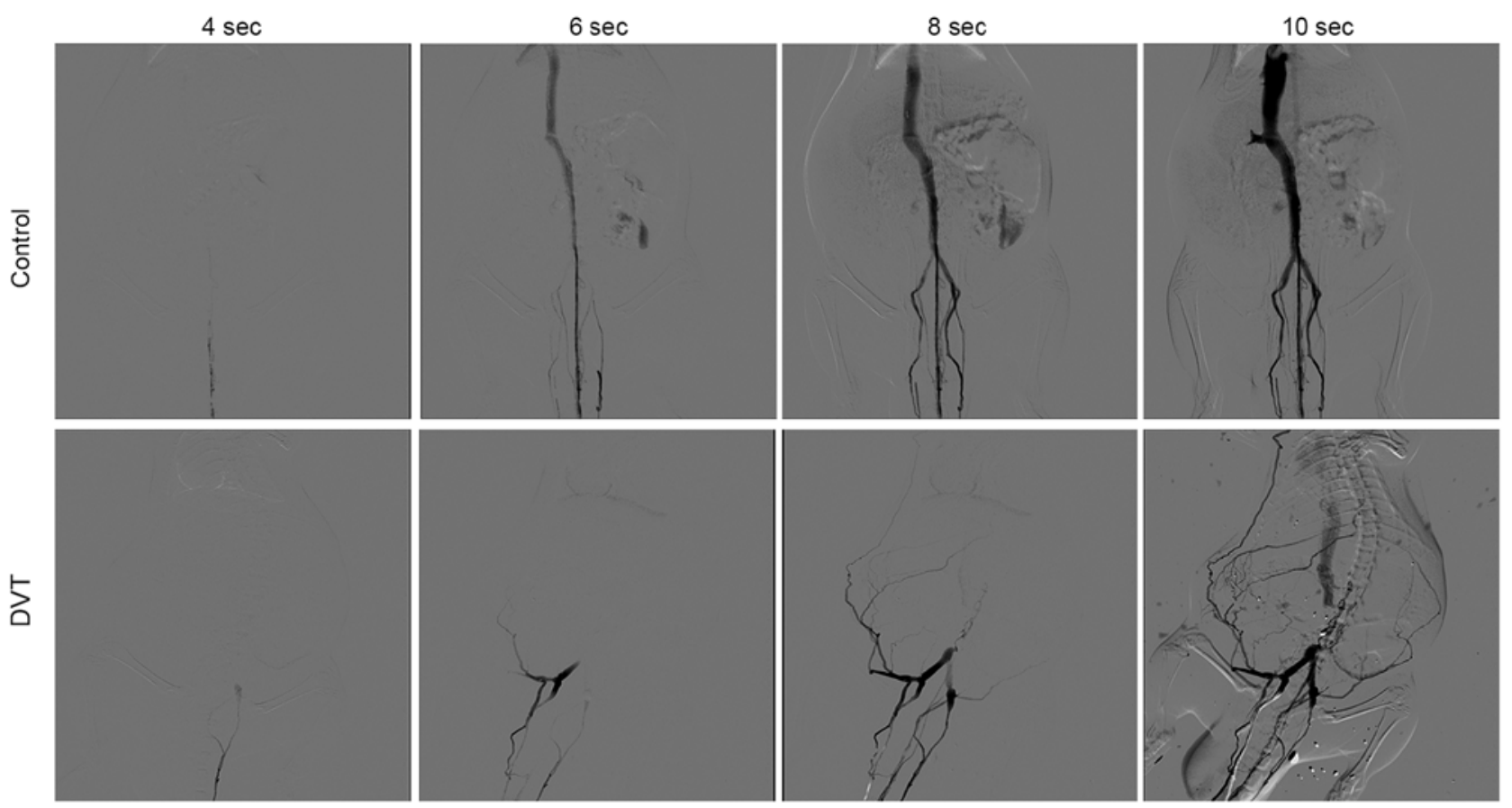

Figure 2. Monitoring inferior vena cava thrombosis using digital subtraction angiography. The abscissa represents different time points after the injection of contrast agent. DVT, deep vein thrombosis.

after the injection of contrast agent. In the control group who did not receive ligation, the IVC was unobstructed and no filling defect and occlusion was observed. In the ligation groups, a partial intraluminal filling defect of the IVC was visualized under the ligation point, with the formation of side branches. The images indicate that only a small amount of contrast agent successfully passed through the occlusion site and reached vessels above the renal vein through side branches. The results demonstrated that the DVT model was successfully established via stenosis of IVC.

Thrombi size progression over time. Using a vernier caliper and electronic balance, the length and weight of thrombi formed at h 1, 4, 6 and 12 and day 1, 3, 7, 14 and 21 were measured after the model was established. A visible thrombus was observed as early as $1 \mathrm{~h}$ after ligation. Thrombus was found only in 4 out of 6 rats at $1 \mathrm{~h}$ and 21 days after modeling. Thrombus weight and length were relatively low in the early stages of modeling
$(1,4,6,12 \mathrm{~h})$ and no significant difference was observed among the four subgroups. The thrombi size was stabilized at a higher level at $24 \mathrm{~h}$, day 3 and 7 after modeling and began to decrease afterwards. However, there was no significant difference between the results of 14 and 21 day $(P>0.05)$. In general, the results suggested that the thrombi size and weight exhibited three distinct periods (1-12, 24 h-day 7 and 14-21), as presented in Fig. 3 .

Histopathological results. The examination of the H\&E-stained thrombi and endothelium sections revealed that no thrombosis and disruption of endothelial cell layer was observed in the control group. Meanwhile, visible thrombus composed of fibrin red blood cells which infiltrated with an abundance of inflammatory cells was identified in the experiment group. The mechanization process (obvious fibrosis hyperplasia was observed around the thrombus) started as early as the first day after modeling, and the completely mechanization was 
A

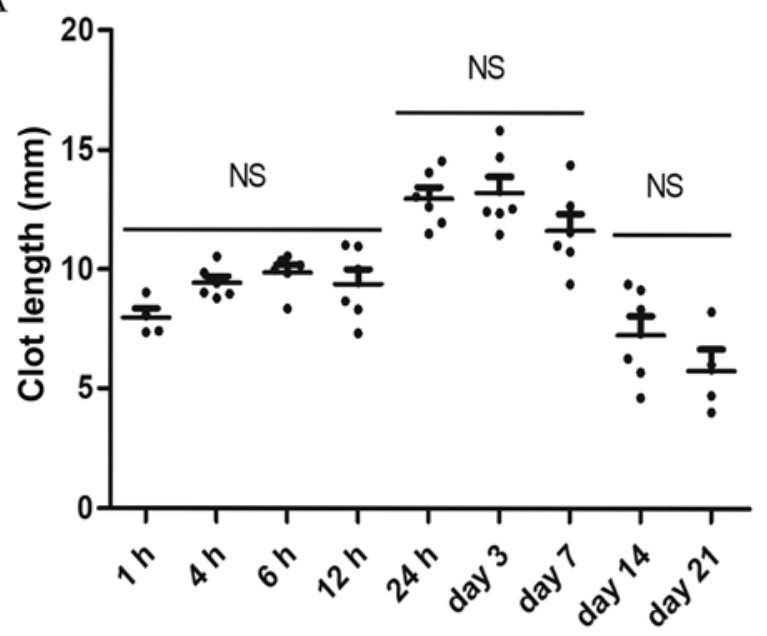

B

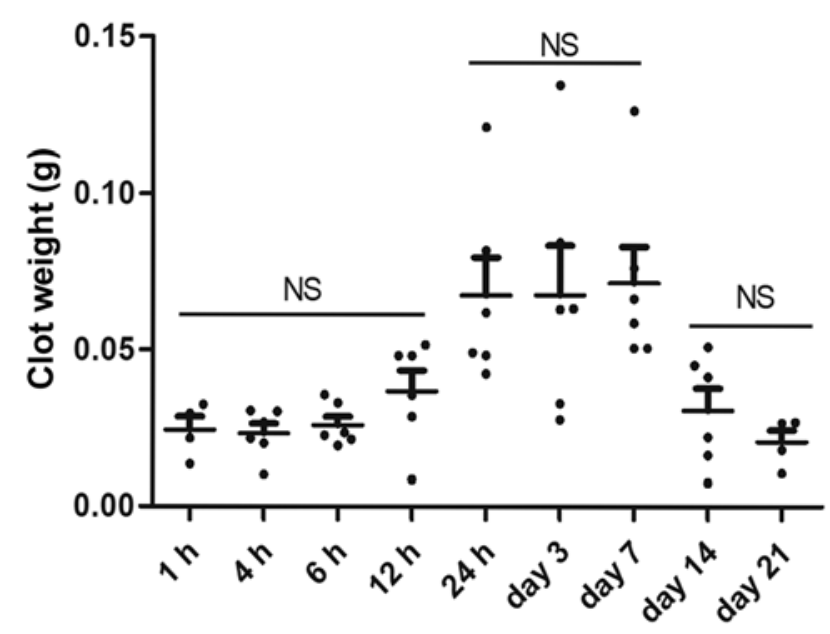

Figure 3. Measurement of thrombus size at different time points. (A) Thrombus length at h 1, 4, 6 and 12 and day 1,3, 7, 14 and 21 post ligation after modeling. (B) Thrombus weight at h 1, 4, 6 and 12 and day 1,3,7,14 and 21 post ligation. Data are presented as mean \pm SEM. $n=6$, thrombus was found only in 4 out of 6 rats at $1 \mathrm{~h}$ and 21 days after modeling. NS, no statistical significance between groups.

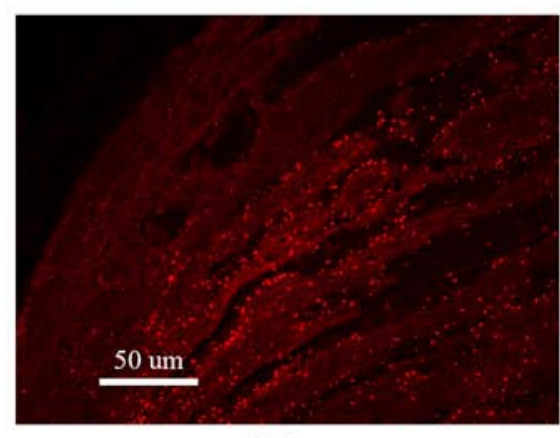

TM

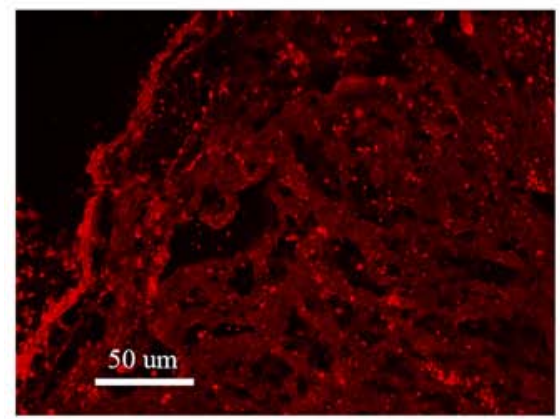

$\mathrm{NF}-\mathrm{kB}$

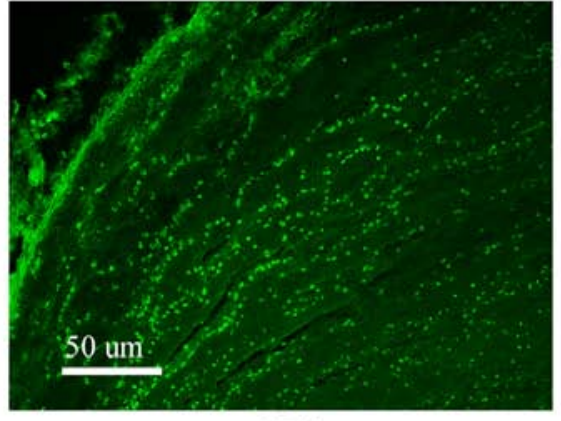

$\mathrm{CD} 34$

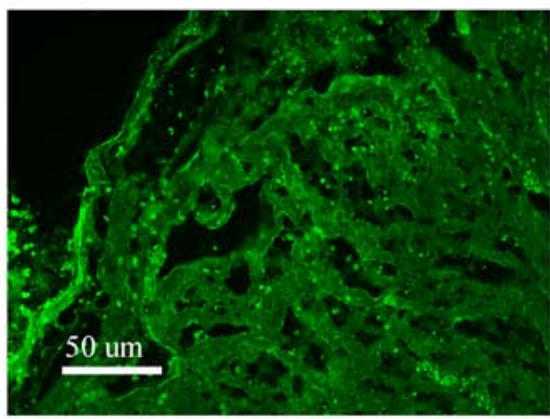

CD34

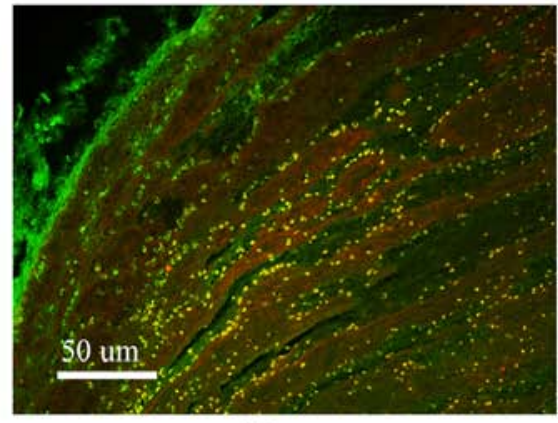

Merge

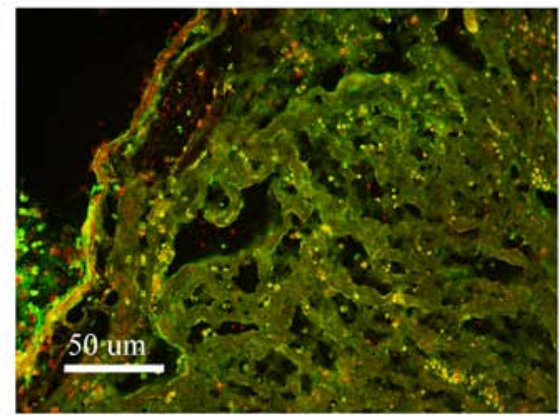

Merge

Figure 4. Immunofluorescence staining presented the locations of TM and p65 in endothelial cells of the DVT group on day 1 post operation. Both TM and p65 were co-labeled with the endothelial cell marker CD34. TM, Thrombomodulin; CD, cluster of differentiation.

observed at about 2 weeks. Organized thrombus was firmly adhered to the blood vessel walls and massive fissures were presented due to the dried thrombus (recanalization). A period of 21 days after modeling, only a small amount of red blood cells and fibrin remained in the inferior vena cava. The representable pathological changes of the thrombus as well as vein wall at various time points were presented in Fig. S2.

Immunofluorescence results. In order to study the distribution of $\mathrm{TM}$ and $\mathrm{NF}-\kappa \mathrm{B}$ (p65), immunofluorescence examination was performed. It was indicated that $\mathrm{TM}$ and $\mathrm{NF}-\kappa \mathrm{B}$ (p65) co-localized within cells expressing CD34. Additional examination further confirmed the co-localization of TM and $\mathrm{NF}-\kappa \mathrm{B}$ (p65) in endothelial cells (Fig. 4).

$T M$ and $N F-\kappa B$ expression over time. In order to elucidate the role and regulatory mechanism of TM during the evolution of DVT, the levels of TM and NF- $\kappa \mathrm{B}$ were monitored and compared with the control group in plasma and thrombi and endothelium tissues at the h 1, 4, 6 and 12 and day 1, $3,7,14$ and 21 post ligation. The results revealed that DVT induced the expression of $\mathrm{TM}$ and $\mathrm{NF}-\kappa \mathrm{B}$ in plasma over time (Fig. 5A and B). The plasma TM levels from 1-12 h post operation $(1 \mathrm{~h}, 18.70 \pm 0.08 \mu \mathrm{g} / \mathrm{l} ; 4 \mathrm{~h}, 18.94 \pm 0.15 \mu \mathrm{g} / \mathrm{l}$; 
A

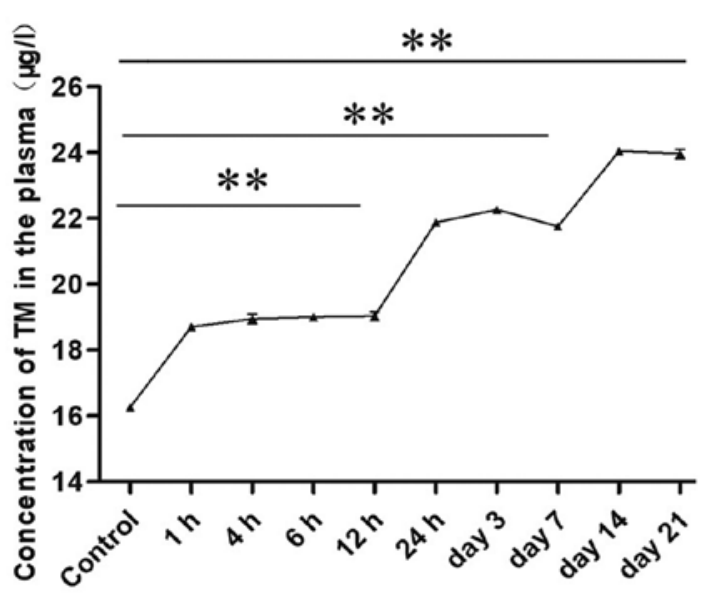

$\mathrm{C}$

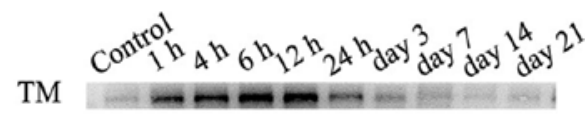

$\beta$-actin

$\mathrm{D}$
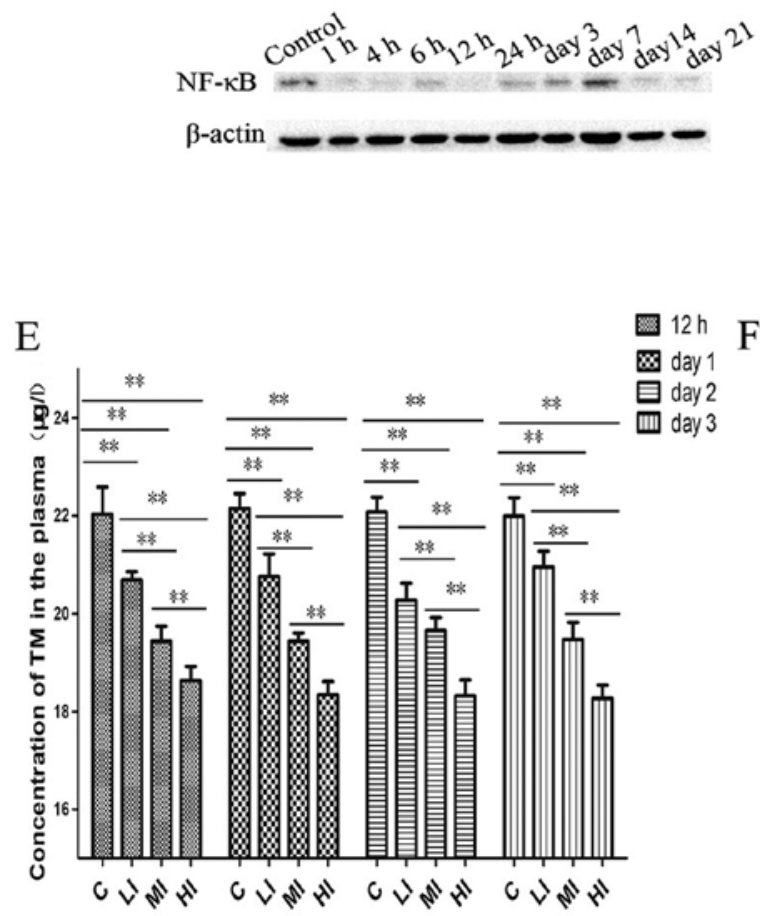

B

ิㅡㄹ
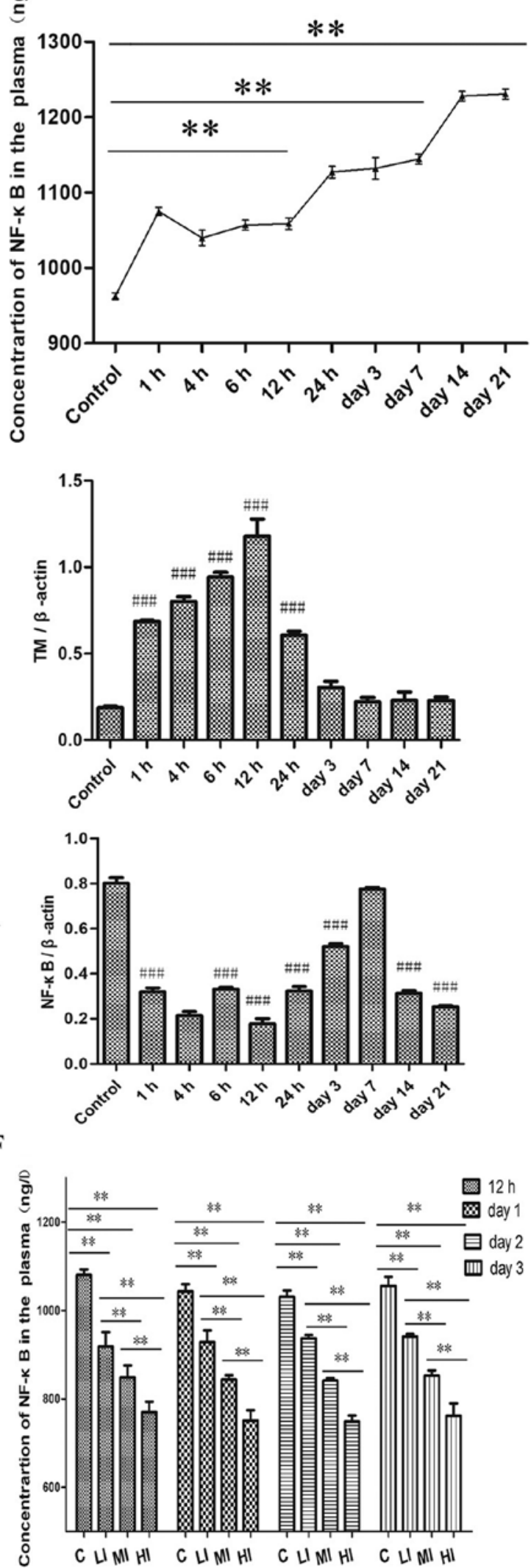

Figure 5. TM and NF- $\mathrm{KB}$ expression over time. (A) The concentration of TM in the plasma during the evolution of DVT. DVT group vs. the control group: ${ }^{*} \mathrm{P}<0.05,{ }^{* *} \mathrm{P}<0.01,{ }^{* * *} \mathrm{P}<0.001$. (B) The plasma level of NF- $\mathrm{kB}$ at different time points after modeling. DVT group vs. the control group. (C) The protein expression changes of TM and (D) NF- $\mathrm{kB}$ in endothelium tissues over time. ${ }^{\#} \mathrm{P}<0.05,{ }^{\# \#} \mathrm{P}<0.01$ and ${ }^{\# \# "} \mathrm{P}<0.001$ vs. the control group. (E) The concentration of TM and (F) NF-kB in the plasma after the administration of PDTC. C, DVT model group (0 mg/kg PDTC); LI, DVT rats treated with $50 \mathrm{mg} / \mathrm{kg}$ PDTC; MI, DVT rats treated with $100 \mathrm{mg} / \mathrm{kg}$ PDTC; HI, DVT rats treated with $150 \mathrm{mg} / \mathrm{kg}$ PDTC. LI, MI, HI vs. the control group; ${ }^{*} \mathrm{P}<0.05,{ }^{* *} \mathrm{P}<0.01$ and ${ }^{* * *} \mathrm{P}<0.001$ as indicated. TM, Thrombomodulin; DVT, deep vein thrombosis; PDTC, pyrrolidine dithiocarbamate. 
$6 \mathrm{~h}, 19.00 \pm 0.09 \mu \mathrm{g} / \mathrm{l} ; 12 \mathrm{~h}, 19.03 \pm 0.13 \mu \mathrm{g} / \mathrm{l}, \mathrm{P}<0.01)$ were increased compared with the control group $(16.26 \pm 0.08 \mu \mathrm{g} / \mathrm{l})$. The plasma TM levels then plateaued during 1 to 7 days after modeling $(24 \mathrm{~h}, 21.88 \pm 0.06 \mu \mathrm{g} / \mathrm{l}$; day 3, 22.26 $\pm 0.09 \mu \mathrm{g} / \mathrm{l}$; day $7,21.75 \pm 0.06 \mu \mathrm{g} / \mathrm{l})$ with a significant difference compared with the control group $(\mathrm{P}<0.01)$. Plasma TM levels then subsequently increased on days 14 to 21 following ligation (day 14 , $24.04 \pm 0.08 \mu \mathrm{g} / 1$; day $21,23.97 \pm 0.14 \mu \mathrm{g} / \mathrm{l}$ ), which was significant when compared with the control group $(\mathrm{P}<0.01)$.

Plasma NF- $\kappa$ B levels exhibited similar trend to plasma TM levels (control, 962.96 $\pm 4.31 \mathrm{ng} / \mathrm{l} ; 1 \mathrm{~h}, 1075.22 \pm 5.23 \mathrm{ng} / \mathrm{l}$; $4 \mathrm{~h}, 1039.99 \pm 10.25 \mathrm{ng} / 1 ; 6 \mathrm{~h}, 1056.73 \pm 6.66 \mathrm{ng} / 1 ; 12 \mathrm{~h}$, $1058.89 \pm 7.64 \mathrm{ng} / \mathrm{l} ; 24 \mathrm{~h}, 1127.41 \pm 7.90 \mathrm{ng} / \mathrm{l} ; 3$ day, $1132.12 \pm 14.42 \mathrm{ng} / 1 ; 7$ day, $1144.41 \pm 6.73 \mathrm{ng} / 1 ; 14$ day, $1228.10 \pm 6.52 \mathrm{ng} / \mathrm{l} ; 21$ day, $1230.68 \pm 6.69 \mathrm{ng} / \mathrm{l})$. The results suggested that thrombi size, TM and NF- $\kappa \mathrm{B}$ expression exhibited three distinct periods (1-12, 24 h-day 7 and 14-21) of markedly different results between periods. Compared with the control group, TM in the vascular endothelium of the DVT group was upregulated up until day 1 post surgery while NF- $\mathrm{B}$ (p65) was downregulated except for day 7, where no significant difference was demonstrated (Fig. 5C and D). The concentrations of TM and NF- $\kappa \mathrm{B}$ in the thrombus were undetectable (data not shown).

Subsequently, PDTC was used as an inhibitor of $\mathrm{NF}-\kappa \mathrm{B}$ and injected into the DVT model rats via the vein tail. The effect of inhibiting NF- $\kappa \mathrm{B}$ activation was confirmed by ELISA and the result revealed that compared with DVT rats that received saline, the plasma levels of TM and $N F-\kappa B$ were downregulated in rats received PDTC treatment in a dose-dependent manner (C vs. LI; C vs. MI; C vs. HI; LI vs. MI; P<0.01; LI vs. HI; MI vs. HI; all, $\mathrm{P}<0.01$ ), as presented in Fig. 5E and F.

\section{Discussion}

In the present study, the level of TM as a marker of vascular endothelial dysfunction during DVT evolution and its possible association with the NF- $\kappa \mathrm{B}$ signaling pathway. A DVT animal model was established using the 'stenosis' method and the levels of TM at 9 different time points were measured during the evolution of DVT. The data demonstrated that plasma TM levels and thrombus size were increased along with the activation of NF- $\mathrm{BB}$ signaling pathway.

DVT formation has been previously thought to be associated with blood stagnancy, hypercoagulability and venous endothelial dysfunction (24). However, there is increasing evidence suggesting that inflammation also serves an important role in the pathogenesis of DVT $(15,25,26)$.

TM is a glycoprotein and serves as a cofactor for thrombin-mediated activation of protein $\mathrm{C}(\mathrm{PC})$, which is a major anticoagulant that downregulates thrombin formation and decreases thrombus formation $(4,10)$. In addition to its anticoagulant activity, studies have revealed that TM exhibits potent anti-inflammatory capabilities via a variety of molecular mechanisms $(4,10)$.

TM exists not only as a cellular membrane bound form but also as a soluble form within plasma (5). It has been previously indicated that increased levels of soluble TM are associated with vascular damage, infection and sepsis (5). In the current study, it was revealed that the levels of soluble TM were increased significantly in patients with DVT compared with healthy controls with an AUC of 0.874 , providing a promising diagnostic method for DVT, as previously reported (6). Consistent with a previous study, the results of the current study also indicated that circulating TM was associated with age (27). Due to the limited sample size in the current study, further investigations should enroll additional patients to assess the levels of TM among different age groups. A previous report revealed that levels of soluble TM were higher in controls compared with patients with DVT (28); however, another studies results were consistent to the current study in which the VTE group exhibited significantly higher levels of soluble TM, and positively correlated with Caprini risk stratification $(6,29)$. The discrepancy among the studies may be due to the different study population and sample size. The population enrolled in the study by Bombeli et al (28) were Swiss $(n=229)$, whereas patients were Chinese in the current study $(n=71)$. Moreover, there were differences in the inclusion and exclusion criteria. The present study included patients with first-onset acute DVT, while Bombeli et al included patients with a history of one or more venous thromboembolic events. Different populations and sample sizes may have affected the results. It has also been previously suggested that TM exerts anti-inflammatory and anti-fiber deposition effects $(4,10)$. As DVT is an inflammatory disease, the anti-inflammatory effects may also significantly increase by upregulating the expression of TM to exert its anti-inflammatory and anti-fiber deposition effects (15). Based on the effects of TM, the levels of TM may increase in patients with DVT.

Many patients with DVT may be asymptomatic, so their optimal diagnosis period may be missed (30). Despite of its low specificity, the level of plasma TM may be used as a potent dynamic monitoring biomarker, reflecting the evolution of DVT. The current study aimed to investigate the dynamic changes of TM during the evolution of DVT. In further studies, the expression of TM in other inflammatory diseases, such as sepsis, inflammatory bowel disease, ulcerative colitis and pneumonia will be further explored to investigate the specificity of TM. In the current study, plasma levels and endothelial expression of TM at different time points were measured during DVT evolution. At the thrombus formation period (1-12 $\mathrm{h}$ after modeling), plasma levels and endothelial expression of TM were increased. It was speculated that the increase of TM level resulted from the initiation of the inflammatory response and endothelial cell damage. Although the expression of TM in the endothelium decreased after $24 \mathrm{~h}$, the expression of TM in the plasma remain increased, indicating that soluble TM in the plasma may be derived from other sources at this period. In line with the results gained in the present study, Sane et al (29) reported that the plasma TM levels of patients with PE were lower in the acute phase compared with the stable phase (seven months later). However, the exact mechanism behind this phenomenon needed to be further evaluated in future studies.

Recombinant human soluble TM (ART-123) has been reported to be a highly effective antithrombotic agent in the prevention of VTE post total hip replacement surgeries (31). Previous studies have reported that increased TM levels indicated worse prognosis of pregnancy-induced hypertension and pre-eclampsia (32). Meanwhile, recombinant-TM improved not only the fetal-placental blood flow but also oxygenation in the 
placenta and fetal brain, making it a possible candidate treatment strategy for pre-eclampsia complications (4). Therefore, it was speculated that the vascular endothelial cell injury and inflammation promoted prothrombotic changes and induced the observed increase in TM secretion. Therefore, TM may not only be a marker for DVT but also be a potential treatment target.

It has been previously indicated that NF- $\kappa \mathrm{B}$ transcription factor p50 is essential for the pathogenesis of DVT, and suggested that specific inhibitors of p50, such as Andro, may be therapeutically valuable for preventing and treating venous thrombosis (33). In order to identify the mechanism of how TM contributes to the pathogenesis of DVT, NF- $\kappa \mathrm{B}$ in plasma and endothelium was investigated In the current study, although the expression of $\mathrm{NF}-\kappa \mathrm{B}$ in the endothelium was decreased in the DVT groups, plasma NF- $\mathrm{BB}$ levels were increased. The increase of NF- $\kappa \mathrm{B}$ levels in plasma was consistent with the results of a previous study (34). Further studies are required to evaluate the mechanism behind the downregulation of $\mathrm{NF}-\kappa \mathrm{B}$ in the endothelium. The current results revealed that the increased secretion of TM during the evolution of DVT was associated with the NF- $\kappa$ B pathway, confirming its significant role in DVT.

Furthermore, the current study attempted to verify the regulatory effect of $\mathrm{NF}-\kappa \mathrm{B}$ on TM via the induction of PDTC, which is a specific $\mathrm{NF}-\kappa \mathrm{B}$ inhibitor that inhibits the activation and nuclear translocation of $N F-\kappa B$ through inhibiting the phosphorylation of $\mathrm{I} \kappa \mathrm{B}(17,18)$. The results of present study revealed that inhibition of $\mathrm{NF}-\kappa \mathrm{B}$ expression by PDTC reduced plasma TM concentration in a dose-dependent manner. Similarly, Deng et al (35) demonstrated in a lipopolysaccharide (LPS)-induced vascular endothelial injury model in human umbilical vein endothelial cells that Puerarin could prevent vascular endothelial injury by suppressing the activation of $\mathrm{NF}-\kappa \mathrm{B}$, and identified that this mechanism was associated with the downregulation of inflammatory factors and coagulation-related factors including TM.

There were some limitations of the current study. Firstly, it should be noted that rat models were used and these may not perfectly recapitulate all human features of DVT. Especially when considering the fact that the animal model was built on healthy animals with no underlying inflammatory or pro-coagulant conditions. Secondly, the changes of $\mathrm{TM}$ and $\mathrm{NF}-\kappa \mathrm{B}$ were not measured in the in endothelium following the administration of PDTC. Thirdly, this was a single-center study with a cohort of a limited number of patients that may not be necessarily be representable to the general population.

In conclusion, the results of the current study suggested that plasma TM levels were increased significantly in patients with DVT. Furthermore, the results of the animal experiment revealed that the increased secretion of TM during the evolution of DVT was associated with the NF- $\kappa \mathrm{B}$ pathway and changed along with thrombus size, indicating that the level of plasma TM may be a potent biomarker for the evolution of DVT.

\section{Acknowledgements}

Not applicable.

\section{Funding}

The current study was supported by the Medical Innovation Team of Jiangsu Province (grant no. Suweikejiao 2017).

\section{Availability of data and materials}

The datasets used and/or analyzed during the current study are available from the corresponding author on reasonable request.

\section{Authors' contributions}

$\mathrm{XC}, \mathrm{BS}$ and $\mathrm{XY}, \mathrm{YZ}$ contributed to the conception and design of the project. SL and DL collected the clinical samples. XC and BS performed all the experiments. XC drafted the manuscript with critical review by BS, XY and YZ. YZ supervised the findings of this work. All authors read and approved the final manuscript.

\section{Ethics approval and consent to participate}

The protocol of the present study was approved by the Ethics Committee of Affiliated Hospital of Nantong University and informed consent was obtained from all participants.

\section{Patient consent for publication}

Not applicable.

\section{Competing interests}

The authors declare that they have no competing interests.

\section{References}

1. Di Nisio M, van Es N and Büller HR: Deep vein thrombosis and pulmonary embolism. Lancet 388: 3060-3073, 2016.

2. Thomas M, Hollingsworth A and Mofidi R: Endovascular management of acute lower limb deep vein thrombosis: A systematic review and meta-analysis. Ann Vasc Surg 58: 363-370, 2019.

3. Galanaud JP, Monreal M and Kahn SR: Epidemiology of the post-thrombotic syndrome. Thromb Res 164: 100-109, 2018.

4. Shin M, Hino H, Tamura M, Ishizuka B, Tanaka M, Suzuki N and Tateda T: Thrombomodulin improves maternal and fetal conditions in an experimental pre-eclampsia rat model. J Obstet Gynaecol Res 40: 1226-1234, 2014.

5. Anastasiou G, Gialeraki A, Merkouri E, Politou M and Travlou A: Thrombomodulin as a regulator of the anticoagulant pathway: Implication in the development of thrombosis. Blood Coagul Fibrinolysis 23: 1-10, 2012.

6. Fu Y, Liu Y, Chen S, Jin Y and Jiang H: The combination of caprini risk assessment scale and thrombotic biomarkers to evaluate the risk of venous thromboembolism in critically ill patients. Medicine (Baltimore) 97: e13232, 2018.

7. Salvagno GL, Pavan C and Lippi G: Rare thrombophilic conditions. Ann Transl Med 6: 342, 2018.

8. Hoesel B and Schmid JA: The complexity of NF- $\kappa$ B signaling in inflammation and cancer. Mol Cancer 12: 86, 2013.

9. Zhang Q, Lenardo MJ and Baltimore D: 30 Years of NF- $\kappa B$ : A blossoming of relevance to human pathobiology. Cell 168: 37-57, 2017.

10. Shirai Y, Uwagawa T, Shiba H, Shimada Y, Horiuchi T, Saito N, Furukawa K, Ohashi T and Yanaga K: Recombinant thrombomodulin suppresses tumor growth of pancreatic cancer by blocking thrombin-induced PAR1 and NF- $\kappa$ B activation. Surgery 161: 1675-1682, 2017.

11. Ma J and Bai J: Protective effects of heparin on endothelial cells in sepsis. Int J Clin Exp Med 8: 5547-5552, 2015. 
12. Wu CT, Chang $\mathrm{YH}$, Lin $\mathrm{P}$, Chen $\mathrm{WC}$ and Chen MF: Thrombomodulin expression regulates tumorigenesis in bladder cancer. BMC Cancer 14: 375, 2014.

13. Menschikowski M,Hagelgans A,TiebelO, Vogel M,Eisenhofer G and Siegert G: Regulation of thrombomodulin expression in prostate cancer cells. Cancer Lett 322: 177-184, 2012.

14. Song D, Ye X, Xu H and Liu SF: Activation of endothelial intrinsic NF-\{kappa\}B pathway impairs protein C anticoagulation mechanism and promotes coagulation in endotoxemic mice. Blood 114: 2521-2529, 2009.

15. Liang W, Wei F, Yang C, Xie F, Shuai XX, Wang M and Yu M: GDF-15 is associated with thrombus burden in patients with deep venous thrombosis. Thromb Res 187: 148-153, 2020.

16. Yao X, Chen W, Liu J, Liu H, Zhan JY, Guan S, Lu Z, Tang P, $\mathrm{Li} \mathrm{P}$ and Lin B: Deep vein thrombosis is modulated by inflammation regulated via sirtuin $1 / \mathrm{NF}-\kappa \mathrm{B}$ signalling pathway in a rat model. Thromb Haemost 119: 421-430, 2019.

17. Qin JD, Cao ZH, Li XF, Kang XL, Xue Y, Li YL, Zhang D, Liu XY and Xue YZ: Effect of ammonium pyrrolidine dithiocarbamate (PDTC) on NF- $\kappa$ B activation and CYP2E1 content of rats with immunological liver injury. Pharm Biol 52: 1460-1466, 2014.

18. Yin J, Wu M, Duan J, Liu G, Cui Z, Zheng J, Chen S, Ren W, Deng J, Tan X, et al: Pyrrolidine dithiocarbamate inhibits NF-KappaB activation and upregulates the expression of Gpx1, Gpx4, occludin, and ZO-1 in DSS-induced colitis. Appl Biochem Biotechnol 177: 1716-1728, 2015.

19. Zhang Z, Hu L, Chen W, Zhou C, Gui G and Lin B: Danhong huayu koufuye prevents deep vein thrombosis through anti-inflammation in rats. J Surg Res 201: 340-347, 2016.

20. Diaz JA, Obi AT, Myers DD Jr, Wrobleski SK, Henke PK, Mackman N and Wakefield TW: Critical review of mouse models of venous thrombosis. Arterioscler Thromb Vasc Biol 32: 556-562, 2012

21. Vukojevic J, Siroglavic M, Kasnik K, Kralj T, Stancic D, Kokot A, Kolaric D, Drmic D, Sever AZ, Barisic I, et al: Rat inferior caval vein (ICV) ligature and particular new insights with the stable gastric pentadecapeptide BPC 157. Vascul Pharmacol 106: $54-66,2018$.

22. Sharma A, Fish BL, Moulder JE, Medhora M, Baker JE, Mader M and Cohen EP: Safety and blood sample volume and quality of a refined retro-orbital bleeding technique in rats using a lateral approach. Lab Anim (NY) 43: 63-66, 2014.

23. Goicoechea M, Cía F, San José C, Asensio A, Emparanza JI, Gil AG, López de Cerain A, Aldazabal P, Azpitarte M, Otaegui D and López de Munain A: Minimizing creatine kinase variability in rats for neuromuscular research purposes. Lab Anim 42: 19-25, 2008.

24. Bagot $\mathrm{CN}$ and Arya R: Virchow and his triad: A question of attribution. Br J Haematol 143: 180-190, 2008.

25. Iba T and Levy JH: Inflammation and thrombosis: Roles of neutrophils, platelets and endothelial cells and their interactions in thrombus formation during sepsis. J Thromb Haemost 16 : 231-241, 2018.
26. Vazquez-Garza E, Jerjes-Sanchez C,Navarrete A, Joya-Harrison J and Rodriguez D: Venous thromboembolism: Thrombosis, inflammation, and immunothrombosis for clinicians. J Thromb Thrombolysis 44: 377-385, 2017.

27. Ochi A, Adachi T, Inokuchi K, Ogawa K, Nakamura Y, Chiba Y, Kawasaki S, Onishi Y, Onuma Y, Munetsugu Y, et al: Effects of aging on the coagulation fibrinolytic system in outpatients of the cardiovascular department. Circ J 80: 2133-2140, 2016.

28. Bombeli T, Jutzi M, De Conno E, Seifert B and Fehr J: In patients with deep-vein thrombosis elevated levels of factor VIII correlate only with von Willebrand factor but not other endothelial cell-derived coagulation and fibrinolysis proteins. Blood Coagul Fibrinolysis 13: 577-581, 2002.

29. Sane M, Granér M, Laukkanen JA, Harjola VP and Mustonen P Plasma levels of haemostatic factors in patients with pulmonary embolism on admission and seven months later. Int $\mathbf{J} \mathrm{Lab}$ Hem 40: 66-71, 2018.

30. Kalayci A, Gibson CM, Chi G, Yee MK, Korjian S, Datta S, Nafee T, Gurin M, Haroian N, Qamar I, et al: Asymptomatic deep vein thrombosis is associated with an increased risk of death: Insights from the APEX trial. Thromb Haemost 118: 2046-2052, 2018.

31. Kearon C, Comp P, Douketis J, Royds R, Yamada K and Gent M: Dose-response study of recombinant human soluble thrombomodulin (ART-123) in the prevention of venous thromboembolism after total hip replacement. J Thromb Haemost 3: 962-968, 2005

32. Ayala-Ramírez P, Buitrago T, Poveda A, Rodríguez JL, Olaya-C M and García-Robles R: Increased tissue factor and thrombomodulin expression and histopathological changes in placentas of pregnancies with preeclampsia. J Neonatal Perinatal Med 9: 31-39, 2016.

33. Li YD, Ye BQ, Zheng SX, Wang JT, Wang JG, Chen M, Liu JG, Pei XH, Wang LJ, Lin ZX, et al: NF-kappaB transcription factor p50 critically regulates tissue factor in deep vein thrombosis. J Biol Chem 284: 4473-4483, 2009.

34. Li G, Zhou R, Zhao X, Liu R and Ye C: Correlation between the expression of IL-18 and deep venous thrombosis. Int J Mol Med 42: 2972, 2018.

35. Deng HF, Wang S, Li L, Zhou Q, Guo WB, Wang XL, Liu MD, Liu K and Xiao XZ: Puerarin prevents vascular endothelial injury through suppression of NF-KB activation in LPS-challenged human umbilical vein endothelial cells. Biomed Pharmacother 104: 261-267, 2018.

This work is licensed under a Creative Commons Attribution-NonCommercial-NoDerivatives 4.0 International (CC BY-NC-ND 4.0) License. 